\title{
Malaysian day-type load forecasting
}

\begin{abstract}
Time series analysis has been applied intensively and sophisticatedly to model and forecast many problems in the biological, physical and environmental phenomena of interest. This fact accounts for the basic engineering problem in forecasting the daily peak system load to use time series analysis. ARMA and REgARMA models are among the times series models considered. ANFIS, a hybrid model from neural network is also discussed as for comparison purposes. The main interest of the forecasts consists of three days up to five days ahead predictions for daily data. The pure autoregressive model with an order 2, or AR (2) with a MAPE value of $1.27 \%$ is found to be an appropriate model for forecasting the Malaysian peak daily load for the 3 days ahead prediction. ANFIS model gives a better MAPE value when weekends' data were excluded. Regression models with ARMA errors are found to be good models for forecasting different day types. The selection of these models is depended on the smallest value of AIC statistic and the forecasting accuracy criteria.
\end{abstract}

Keyword: ARMA; ANFIS; RegARMA; MAPE; Load forecasting 